\title{
Pyridoxol metabolism in vitamin B6-responsive convulsions of early infancy
}

\author{
A. HEELEY, ${ }^{*}$ R. J. P. PUGH, $\dagger$ BARBARA E. CLAYTON, JEAN SHEPHERD, AND \\ J. WILSON
}

From the Peterborough District Hospital, Hull Royal Infirmary, and The Hospital for Sick Children, Great Ormond Street, London

SUMMARY As there is uncertainty about the nature of the metabolic defect in vitamin B6responsive convulsions, certain aspects of pyridoxol metabolism were studied in 3 patients who were believed on clinical grounds to have the condition. The findings were compared with those in healthy children and adults, and in children with mental handicap. The magnitude of the initial rise and the subsequent fall in plasma pyridoxal phosphate (PALP) concentrations after a load of pyridoxol suggested that the vitamin B6-responsive patients were able to synthesise PALP normally but were unable to maintain the prolonged high levels normally found in plasma. The urinary excretion of 4-pyridoxic acid was within normal limits, but the excretion of pyridoxol after the load was raised. It is suggested that there may be an instability of the PALP-albumin complex in this condition. Some of the biochemical features were also observed in an infant presenting with convulsions soon after birth but without evidence of clinical B6-dependency.

Vitamin B6-responsive convulsions of early infancy were first described by Hunt et al. (1954). Patients reported subsequently suggest that an autosomal recessive gene is responsible for the clinical phenotype, the principal feature of which is tonic-clonic convulsions appearing before or shortly after birth. The convulsive activity in these infants responds dramatically when pyridoxol (often referred to as pyridoxine) is administered orally in amounts which may vary from 2 to $80 \mathrm{mg}$ or more daily. The usual anticonvulsant drugs have not proved very effective in controlling the fits.

Routine biochemical investigations show no abnormality in the patients, and sensitive indicators of the vitamin B6 cofactor (pyridoxal-5'-phosphate) activity-such as the tryptophan load test (Coursin, 1964) and erythrocyte aspartate aminotransferase cofactor activation (Hamfelt, 1966)-have invariably given normal results. The specific investigation of pyridoxol metabolism has been the subject of two reports (Scriver and Cullen, 1965; Gentz et al., 1967) and in both it was concluded that no disorder

Received 23 September 1977

Present addresses:

* Department of Clinical Chemistry, Peterborough District Hospital, Thorpe Road, Peterborough PE3 6DA.

†Department of Paediatrics, Hull Royal Infirmary, Anlaby

Road, Hull HU33 J2. of systemic vitamin B6 metabolism existed in this clinical condition. It has since been inferred that the underlying metabolic defect involves a specific cerebral enzyme with a requirement for pyridoxal phosphate. Thus, the genetic defect would be manifest in an altered apo-enzyme structure which affected its binding capacity for the cofactor, a situation analogous to several other well-documented inborn errors of metabolism (Mudd, 1971). The pyridoxal phosphate-requiring enzyme, glutamic acid decarboxylase, with its role in the synthesis of $\gamma$-amino-butyric acid, a putative neuroinhibitory transmitter substance, has therefore been implicated (Scriver and Whelan, 1969). There has been no experimental evidence that unequivocally establishes an inborn error of metabolism of this particular enzyme as the primary cause of vitamin B6responsive convulsions of early infancy.

Early reports of the clinical condition described abnormalities in the urinary excretion of pyridoxol and its metabolites (Coursin, 1960; Scriver, 1960), although this was not confirmed in later investigations on older children (Scriver and Cullen, 1965; Gentz et al., 1967). In view of the uncertainty about the nature of the metabolic defect in this neurological disorder it was considered appropriate to re-examine certain aspects of pyridoxol metabolism in healthy children and adults, in sick children, and 
in 4 children considered to have vitamin B6responsive convulsions.

Cases 1 to 3 were the subject of an earlier study on EEG changes in this condition (Willey, 1972). Although Case 4 was manifestly not pyridoxolsensitive in the strict sense, the early onset of fits was typical as was the lack of response to the late administration of pyridoxol.

\section{Subjects}

Studies were made on 3 patients who were believed clinically to have no abnormality of pyridoxol metabolism, and on a 4th infant who had early onset of fits but was not clinically vitamin B6-dependent. Results were compared with those obtained in 3 groups of subjects.

Group A. 12 boys and 7 girls aged 3 weeks to $4 \frac{1}{2}$ years were inpatients at The Hospital for Sick Children and were being investigated for the cause of mental handicap. Their final diagnoses included congenital cerebral malformations, goitrous cretinism, spastic quadriplegia, myoclonic epilepsy, Menkes's disease, and autism. In several patients no definitive diagnosis was made; most presented with convulsions and were receiving anticonvulsant drugs as part of their treatment. Analyses were performed on blood or urine or on both.

Group B. 6 boys and 5 girls aged $2 \frac{1}{2}$ to 10 years were healthy children. Analyses were made on urine only, as no blood could be taken.

Group C. 9 women and 5 men aged 20 to 42 years were healthy adults. Analyses were performed on blood or urine or on both.

\section{Case reports}

Case 1. A term baby girl born normally weighing $4.1 \mathrm{~kg}$ and initially robust, became distressed by 14 hours with shallow breathing and abdominal distension. Almost continuous convulsions began after 24 hours and increased in frequency and violence in spite of paraldehyde and diazepam, but were suppressed at 40 hours by the intramuscular injection of pyridoxol $25 \mathrm{mg}$ repeated 12-hourly. Pronounced changes in muscle tone were observed in relation to pyridoxol administration, with floppiness and irritability alternating with normality.

The child's mother recalled frequent painful episodes during the last trimester of pregnancy, when the fetus had trembled rhythmically for up to half-an-hour. A previous infant born 2 years before had behaved similarly and died after convulsions aged 2 days: necropsy had shown nothing of significance. A diagnosis of pyridoxol-dependent epilepsy seemed likely in retrospect.

The patient progressed at home with periods of trembling, variations in muscle tone, and occasional convulsions until the pyridoxol, $20 \mathrm{mg}$, was increased from 12-hourly to 8-hourly. At 0.92 year, her dependence upon vitamin B6 was challenged and resulted in confusion and hypotonicity after 48 hours followed by a generalised convulsion after $\mathbf{7 2}$ hours, again suppressed by pyridoxol. Irritability and short convulsions at $1 \frac{1}{2}$ years prompted a further increase in dosage to $40 \mathrm{mg}$ 8-hourly with incomplete control, until at $2 \frac{1}{2}$ years during a further deterioration $80 \mathrm{mg}$ 8-hourly was begun, but increased confusion and clumsiness followed and a reduction to $60 \mathrm{mg} 8$-hourly proved optimal.

Her developmental progress was watched anxiously. She could sit indefinitely by 0.9 year, moving in this position but not crawling during the second year, and walking independently but insecurely at 2.25 years. Clumsiness has persisted and affects manipulation equally. Periods of apathy and confusion persist, but to a diminishing degree with variation in muscle tone, calf muscle spasms, and toe walking associated with explosive deep reflexes. Developmental assessment at intervals indicated a reduced intelligence within the mildly mentallyhandicapped range and a specific problem in motor co-ordination. Her EEG records were consistently normal during the intervals between convulsions, provided pyridoxol supplementation was maintained. After 2 years at the local school she was transferred for special education to a school for the mildly mentally handicapped with an IQ on the Wechsler preschool and primary scale of 68 (verbal scale 64 , performance scale 80 ).

Case 2. This infant, the only child of nonconsanguineous healthy parents, had a normal birth in hospital 10 days before the expected date and weighed $2.8 \mathrm{~kg}$ after an unremarkable pregnancy. Her condition at birth was excellent, but $2 \frac{1}{2}$ hours later she was moved to the special baby nursery agitated and trembling. Generalised convulsions began shortly afterwards and were soon violent and almost continuous. Pyridoxol, $50 \mathrm{mg}$, was given intramuscularly at 4 hours of age with complete suppression of convulsions within 10 minutes; this was sustained by $20 \mathrm{mg}$ given 12-hourly by mouth for the next week. The diagnosis of dependency was then challenged by stopping the pyridoxol. After several days of irritability convulsions were resumed on day 5 and were again suppressed promptly with pyridoxol. This whole sequence was then repeated with EEG monitoring and the dependency confirmed without any doubt. Similar observations 
were made at 2.5 and 4 years, confirming that there had been no change in her dependence on pyridoxol supplementation. It was noted that after stopping the maintenance dose, convulsions, preceded by a period of weakness and confusion, resulted in 4 or 5 days and that several days of neurological abnormality persisted after the resumption of pyridoxol.

Motor development has been marginally slow with walking by 1.42 years, the gait remaining insecure and quick to tire. At 5 years, she was socially acceptable as a school entrant, but remained a dreamy observer and by 6 was transferred for special educational facilities in a school for the mildly mentally handicapped. At that time her motor proficiency, drawing, and language development were at the 4-year level and her concentration span was short.

Case 3. The second child of healthy nonconsanguineous parents was born in a maternity home at term and weighed $3.2 \mathrm{~kg}$. Pregnancy had been complicated by unprovoked periodic hammering of the uterus during the last trimester, interpreted retrospectively as intrauterine convulsions. Although robust at birth he was covered in meconium and needed some routine cleaning of the airways. Breathing distress was reported at 2 hours and generalised convulsions supervened at 4 hours when he was transferred to the district hospital. Routine investigations of CSF and blood were normal, and standard anticonvulsant treatment was ineffective. By 17 hours the convulsions were violent and almost continuous with limbs extended and head thrown back. Pyridoxol, $20 \mathrm{mg}$, was then given intramuscularly and after 15 to 20 minutes the fits stopped, the infant becoming limp and apnoeic. Ventilation with oxygen after tracheal intubation was necessary for an hour before regular breathing was resumed.

During the next 48 hours while taking pyridoxol $20 \mathrm{mg}$ 12-hourly by mouth, the infant gradually became normally strong and looked remarkably improved. Pyridoxol supplementation was stopped at the end of 3 days as its relevance seemed in doubt, but after another 4 days the infant again became limp and on the 10th day of life convulsions were resumed and continued during the next week, giving the impression of intrapartum brain damage until pyridoxol $20 \mathrm{mg}$ 12-hourly was eventually resumed at age 17 days. There was again a remarkable change from tonic convulsions and rigidity to hypotonicity with abdominal distension and respiratory depression, but there were no further fits.

While monitoring with EEG, a month later pyridoxol was stopped in order to challenge dependency, but within 4 days sustained convulsions were resumed with explosive deep reflex responses and sustained ankle clonus. The resumption of 20 mg pyridoxol 12-hourly was effective but when the dose was reduced at 2.42 years of age to $10 \mathrm{mg}$ 12-hourly, confusion, restlessness, and a state of profound hypotonia occurred within a week. This resolved gradually as he was given more pyridoxol and he remains well at $6 \frac{3}{4}$ years on $20 \mathrm{mg}$ pyridoxol 12-hourly by mouth, and shows little clinical variation from day to day.

Sitting was achieved at one year with independent walking by 2 years. Large movements were clumsy until he entered school and at 6 years the motor proficiency (Stott) was at the 4-year level with representational drawing and speech less well advanced. The child is awaiting transfer for special schooling appropriate for the mildly mentally handicapped.

In addition to these 3 cases in which there was clear evidence of vitamin B6-dependency, we include details of an infant presenting with convulsions soon after birth, who, although lacking clinical evidence of vitamin B6-dependency, shared with Cases 1-3, certain biochemical features.

Case 4. This baby girl was the first child of healthy nonconsanguineous parents. The birthweight was $3.4 \mathrm{~kg}$ and head circumference $34 \mathrm{~cm}$. Fetal movements had been normal, but twitching of the left eyelid was observed within a few hours of delivery and major fits were first recognised on the 2nd day. They continued thereafter daily with a frequency which seemed to vary little with conventional anticonvulsants.

When investigated at 0.25 year, the child was socially responsive, but generally hypotonic, with a head circumference of $36 \mathrm{~cm}$. An EEG showed a marked abnormality over the right frontocentral region with frequent focal discharges. Pyridoxol, $300 \mathrm{mg}$, was given by mouth daily in addition to phenobarbitone and phenytoin, but a repeat EEG after 4 days was, if anything, worse than the first, with multifocal discharges. Fit control remained poor in spite of increasing the pyridoxol dosage to $600 \mathrm{mg}$ daily, although there was an impression of transient improvement in fit frequency for 24 hours. When reinvestigated at 2 years, she was severely subnormal and generally hypotonic. Major fits with predominantly left-sided involvement continued several times daily and head circumference was below the 3rd centile.

\section{Methods}

Plasma pyridoxal phosphate (PALP) was measured by the method of Chabner and Livingstone (1970). 
4-Pyridoxic acid (4-PA) was measured in urine by the method of Reddy et al. (1958) as modified by Storvick et al. (1964). After urinary pyridoxol (POL) and pyridoxal (PAL) had been separated from 4-PA by the ion-exchange technique of Storvick $\mathrm{et}$ al. (1964), POL and PAL were measured fluorimetrically using the procedures described by Storvick et al. (1964) and Bonavita (1960) respectively.

Administration of pyridoxol. Pyridoxol hydrochloride in a dosage of $5 \mathrm{mg} / \mathrm{kg}$ was dissolved in fruit juice or milk and taken orally at breakfast by adults and older children, or administered with the morning feed to infants.

Collection of blood and urine. Capillary blood (1-2 $\mathrm{ml}$ ) was collected by heel or finger prick (for timing, see individual investigations) into tubes containing EDTA as anticoagulant. The tubes were wrapped in metal foil to exclude light and placed on ice until they were transferred to the laboratory where the plasma was promptly separated in a refrigerated centrifuge and stored in a liquid nitrogen refrigerator $\left(-70^{\circ} \mathrm{C}\right)$ until analysed within 2 weeks of collection. Urine was collected for 7 to 8 hours from the time pyridoxol was administered. Timing was approximate as no child was catheterised. Specimens were preserved with 1 to 2 drops $10 \%$ (w/v) thiomersal, protected from light and stored at $-20^{\circ} \mathrm{C}$ until analysed.

Preliminary studies had shown that the excretion of the major metabolite (4-PA) occurred maximally 3 to 5 hours after the ingestion of pyridoxol and this was followed by a sharp decline so that only 2 to $3 \%$ of the administered vitamin was excreted as 4-PA between the 7th and 8th hours. The excretion of POL occurred maximally within 2 hours of its ingestion and the amount excreted between the 7th and 8th hour was of the order of $0.5 \%$. Urine was therefore not collected 8 hours after administration of pyridoxol. Urinary excretions of POL and its metabolites in 8 hours were expressed as percentages (molar) of the pyridoxol hydrochloride ingested.

\section{Investigations and results}

Urinary excretion of pyridoxol and its metabolites after oral pyridoxol was studied in groups A, B, and $\mathrm{C}$, and in the 4 patients. Three of the patients (Cases 1,2 , and 3) had been prescribed large amounts of the vitamin daily from early infancy, but had received none for a period of 16 to 24 hours before the test as the last dose of the previous day was omitted. Urine voided immediately before the test contained amounts of 4-PA, PAL, and POL which were not significantly different from those in baseline specimens of the control subjects.

Results are given in Table 1 . Healthy adults and children gave similar results. Children in group A had a reduced excretion of POL and an increased excretion of PAL compared with those in group B, but their mean age was only 2 compared with $6 \frac{1}{2}$ years for healthy children. However, it is conceivable that these differences in the metabolism of vitamin B6 related to their general clinical condition. Of the 4 patients, the excretion of POL in 3 (Cases 1, 2, and 4) was greater than the mean for groups A, B, and $\mathrm{C}$ by more than 3 SDs. The excretion of the metabolites 4-PA and PAL was not significantly different from the values observed for any of the control groups.

PALP was measured immediately before (fasting), and 1,2 , and 4 hours after the ingestion of the pyridoxol load. The levels attained at these times in 14 healthy adults and in 8 children are shown in Table 2. A mean 10-fold increase was observed within 4 hours of the ingestion of the vitamin. In each individual the plasma PALP concentration was greater at 4 hours than at 2 .

To assess how long the increase in plasma PALP persisted, 6 adults were tested 24 hours after the load, and in each case the plasma PALP concentration was greater than that observed at 4 hours. At 48 hours all subjects had a PALP concentration which was less than the 4-hour value (Table 3). Three children were studied from group A. Levels were declining by 24 hours.

Table 1 Urinary excretion of pyridoxol and metabolites (as \% of ingested pyridoxol) by sick and healthy children and by adults (mean $\pm 1 S D)$ and Cases 1-4

\begin{tabular}{|c|c|c|c|c|c|c|}
\hline Group & Subjects & No. & Age (years) & 4-Pyridoxic acid & Pyridoxal & Pyridoxol \\
\hline $\begin{array}{l}\text { A } \\
\mathbf{B} \\
\mathbf{C}\end{array}$ & $\begin{array}{l}\text { Children } \\
\text { Sick } \\
\text { Healthy } \\
\text { Adults }\end{array}$ & $\begin{array}{l}11 \\
11 \\
11\end{array}$ & $\begin{array}{l}2 \text { (mean) } \\
6 \cdot 5 \text { (mean) } \\
30 \text { (mean) }\end{array}$ & $\begin{array}{l}22 \cdot 1 \pm 6 \cdot 4 \\
28 \cdot 5 \pm 6 \cdot 0 \\
31 \cdot 1 \pm 3 \cdot 5\end{array}$ & $\begin{array}{l}4 \cdot 7 \pm 2 \cdot 6^{*} \\
2 \cdot 2 \pm 0 \cdot 6 \\
2 \cdot 0 \pm 0 \cdot 5\end{array}$ & $\begin{array}{r}7 \cdot 9 \pm 2 \cdot 8 \dagger \\
13 \cdot 1 \pm 2 \cdot 7 \\
12 \cdot 3 \pm 1 \cdot 9\end{array}$ \\
\hline $\begin{array}{r}\text { Vitamin } \\
\text { Case } \\
\text { Case } 4\end{array}$ & $\begin{array}{l}\text { n6-respons } \\
1 \\
2 \\
3\end{array}$ & & $\begin{array}{l}1 \cdot 66 \\
0.83 \\
1 \cdot 25 \\
0.33\end{array}$ & $\begin{array}{l}11 \cdot 5 \\
21 \cdot 6 \\
14 \cdot 1 \\
32 \cdot 5\end{array}$ & $\begin{array}{l}2 \cdot 2 \\
4 \cdot 8 \\
1 \cdot 6 \\
\text { Not done }\end{array}$ & $\begin{array}{l}27 \cdot 0 \\
21 \cdot 2 \\
13 \cdot 5 \\
27 \cdot 2\end{array}$ \\
\hline
\end{tabular}

Compared with group B: *significant at 0.01 level; †significant at 0.001 level. 
798 Heeley, Pugh, Clayton, Shepherd, and Wilson

Table 2 Plasma pyridoxal phosphate (nmol/l) after administration of pyridoxol $\mathrm{HCl}(5 \mathrm{mg} / \mathrm{kg})$ to sick children and adults

\begin{tabular}{lllr}
\hline Group & Fasting & \multicolumn{2}{l}{ Plasma pyridoxal phosphate (mean \pm SD) } \\
\cline { 3 - 4 } & & 1 hour & 2 hours \\
\hline A Sick children* & $58 \pm 21$ & $344 \pm 111$ & $421 \pm 125$ \\
C Adults & $50 \pm 13$ & $317 \pm 92$ & $417 \pm 126$ \\
\hline
\end{tabular}

*5 boys, 3 girls; age 0.25-5 years; +9 men, 5 women; age $20-42$ years.

Table 3 Plasma pyridoxal phosphate (nmol/l) after administration of pyridoxol $\mathrm{HCl}(5 \mathrm{mg} / \mathrm{kg})$ to sick children and adults

\begin{tabular}{|c|c|c|c|c|}
\hline \multirow[t]{2}{*}{ Group } & \multirow[t]{2}{*}{ Subjects } & \multicolumn{3}{|c|}{ Plasma pyridoxal phosphate } \\
\hline & & 4 hours & 24 hours & 48 hours \\
\hline $\mathbf{A}$ & $\begin{array}{l}\text { Sick children }(n=3) \\
1.08 \text { years } \\
1.33 " \\
1.25 "\end{array}$ & $\begin{array}{l}498 \\
660 \\
356\end{array}$ & $\begin{array}{l}291 \\
489 \\
227\end{array}$ & \\
\hline
\end{tabular}

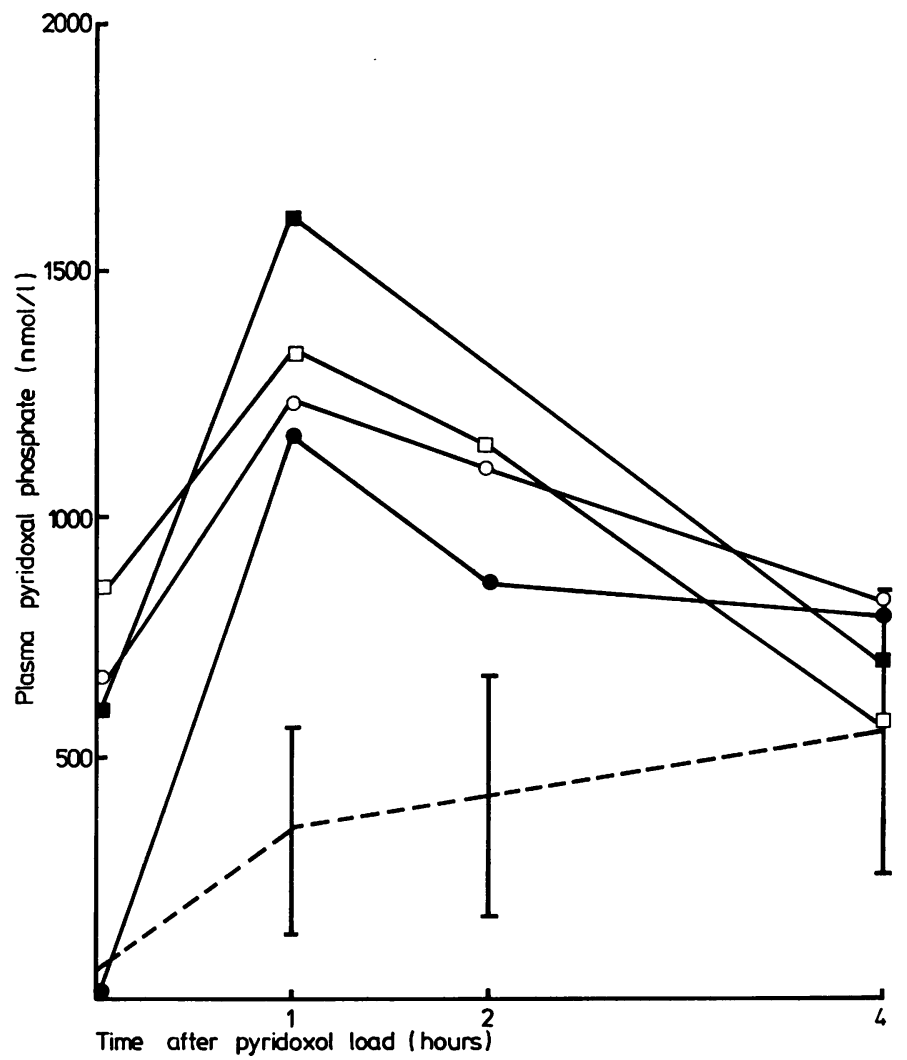

Patients

Case 1 (age 1.66 years)
Case 2 (age 0.83 year)

O Case 3 (age 1.25 years)

- Case 4 (age 0.33 year)

Control children group A (mean \pm 2 SD) $\mathrm{I}---\mathrm{I}$

$(n=8$, mean age 2 years).

Fig. 1 Plasma pyridoxal phosphate

(nmol/l) fasting, and 1, 2, and 4 hours after administration of pyridoxol $\mathrm{HCl}$ orally $(5 \mathrm{mg} / \mathrm{kg})$. 
The vitamin B6-responsive patients (Cases 1-3) and Case 4 exhibited an entirely different response to the pyridoxol load. The changes in PALP concentrations during the 4-hour period are shown in Fig. 1. There was a rapid increase in PALP within the first hour, and during the next 3 hours the concentrations decreased. Cases 1 to 3 had been receiving pyridoxol therapeutically and their plasma specimens immediately before the oral load contained large amounts of PALP.

To assess whether the abnormal responses to the load reflected a 'saturation' phenomenon caused by the administration of large amounts of pyridoxol for many months, two normal adults were given the oral load after receiving $30 \mathrm{mg}$ pyridoxol daily for 10 days. During the 4-hour period (Fig. 2), plasma PALP levels increased in spite of the high baseline values which were similar to those found in the patients. In addition, Case 4 who had not received pyridoxol for therapeutic purposes immediately before the load, showed the same abnormal response in plasma PALP levels as the other 3 patients. Her concentration of fasting plasma PALP was $4 \mathrm{nmol} / \mathrm{l}$ which was less than the mean for the control children by more than 2 SDs. Particularly apparent in this patient (Fig. 1) is the anomalous increase in PALP during the first hour after the oral load.

Cases 1 to 4 were further investigated when 3 of the patients were 1.03 years older and the 4th (Case 1) 1.67 years older. The results are shown in Table 4 and Fig. 3. On this occasion the response to the pyridoxol load was normal, although Case 4 who, as previously, was not receiving additional pyridoxol at the time of admission, had a low fasting plasma PALP $(8 \mathrm{nmol} / \mathrm{l})$. In this patient also, the plasma PALP at 1, 2, and 4 hours remained 2 SDs below the mean for the controls, although the levels did continue to rise.

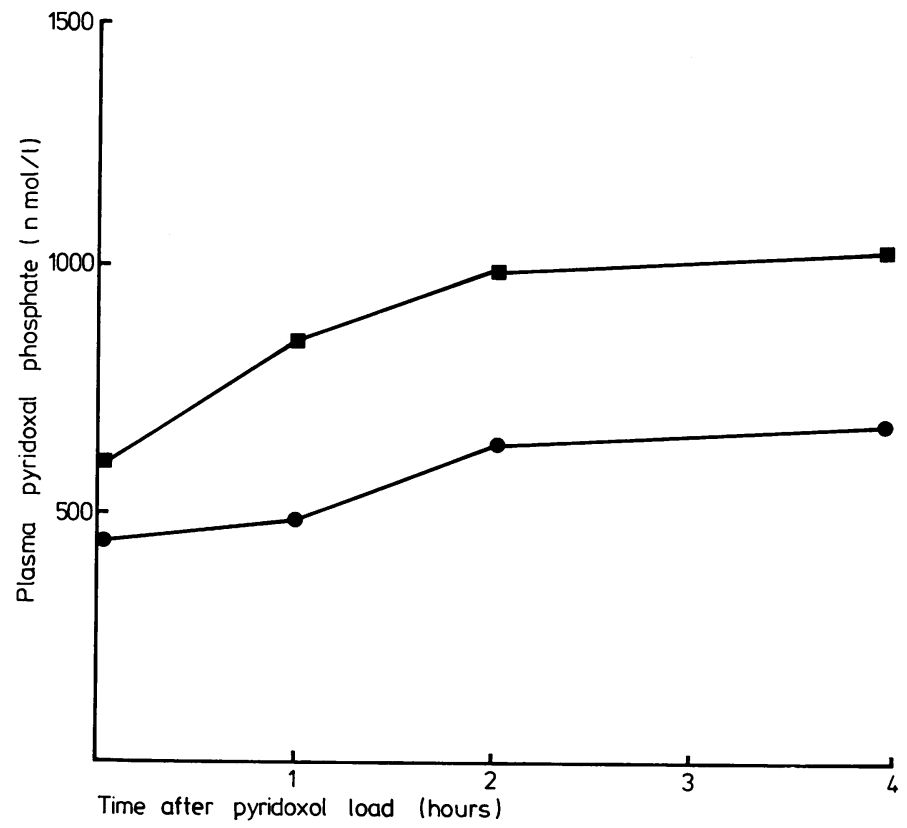

Fig. 2 Plasma pyridoxal phosphate (nmol/l) fasting, and 1, 2, and 4 hours after administration of pyridoxol $\mathrm{HCl}$ orally $(5 \mathrm{mg} / \mathrm{kg})$ in 2 normal adults who had received pyridoxol $\mathrm{HCl}$ (30 mg) daily for 10 days.

Table 4 Urinary excretion of pyridoxol and metabolites (as \% of ingested pyridoxol HCl) by control subjects and Cases $1-4$ (mean $\pm 1 S D)$

\begin{tabular}{|c|c|c|c|c|c|c|}
\hline Group & Subjects & No. & Age (years) & 4-Pyridoxic acid & Pyridoxal & Pyridoxol \\
\hline $\mathbf{A}$ & Sick children & 11 & 2 (mean) & $22 \cdot 1 \pm 6 \cdot 4$ & $4 \cdot 7 \pm 2 \cdot 6$ & $7 \cdot 9 \pm 2 \cdot 8$ \\
\hline \multicolumn{7}{|c|}{ Vitamin B6-responsive patients } \\
\hline & Case 1 & & 2.69 & $27 \cdot 0$ & 3.0 & $7 \cdot 6$ \\
\hline & 2 & & 1.86 & $33 \cdot 8$ & $3 \cdot 1$ & $8 \cdot 8$ \\
\hline & 3 & & $2 \cdot 28$ & 15.9 & $3 \cdot 1$ & 9.0 \\
\hline Case 4 & & & 2.00 & 14.6 & 2.0 & $12 \cdot 0$ \\
\hline
\end{tabular}




\section{Discussion}

Cases 1 to 3 clearly show vitamin B6-dependency, the clinical responses to administration and withdrawal of the vitamin being particularly striking. In 2 of them, there was also a history of fetal fits in utero. The evidence on clinical grounds for B6dependency in Case 4 is absent, but the apparent failure of the vitamin could be caused by delay in treatment started only at 3 months. In this case, damage could have occurred so that epileptic foci were well established and active, even though B6 was adequately supplemented later. Case 4 shared with Cases 1 to 3 certain biochemical features.

There is general agreement that the major route of synthesis for PALP proceeds according to the sequence (1) POL $\rightarrow$ pyridoxol phosphate (POLP) $\rightarrow$ PALP and to a much lesser extent synthesis can take place via (2) POL $\rightarrow$ PAL $\rightarrow$ PALP (Johansson et al., 1968, 1974; Colombini and McCoy, 1970; Tiselius, 1973). In man 4-PA is the main urinary excretion product of vitamin B6 metabolism (Rabinowitz and Snell, 1949). 4-PA is formed by the action of an aldehyde oxidase on PAL. The latter is not only formed by reaction sequence (2) but also by the action of phosphatase on PALP.

There is evidence to suggest that the constant supply of cofactor PALP required for metabolic purposes would not be adequately met by the infrequent ingestion of dietary vitamin, but would be more effectively provided from a storage pool (Johansson et al., 1966). Moreover, it appears that the brain with its large metabolic requirement for the cofactor is supplied by an extracerebral storage compartment (Colombini and McCoy, 1970; Tiselius, 1973). Transport from such a site would require that the vitamin was in a metabolically inert form. It is likely that plasma albumin, which possesses a binding site with strict steric requirements and a strong affinity for PALP, could fulfil this role (Dempsey and Christensen, 1962; Anderson et al., 1971). Hepatocyte membranes seem to be unique in possessing a mechanism for the transport of PALP out of the cell, possibly already bound to newly synthesised albumin (Lumeng et al., 1974). The PALP-albumin complex is slowly hydrolysed by the phosphatase of plasma membranes releasing PAL which, because it does not bind to protein, would be readily absorbed and rephosphorylated intracellularly.

In the present investigations, the magnitude of the initial rise and the subsequent fall in plasma PALP concentrations, suggest that the vitamin B6responsive patients were able to synthesise PALP normally, but were unable to maintain the prolonged high levels normally found in plasma after pyridoxol loading. Such findings might be explained by an instability of the PALP-albumin complex or,

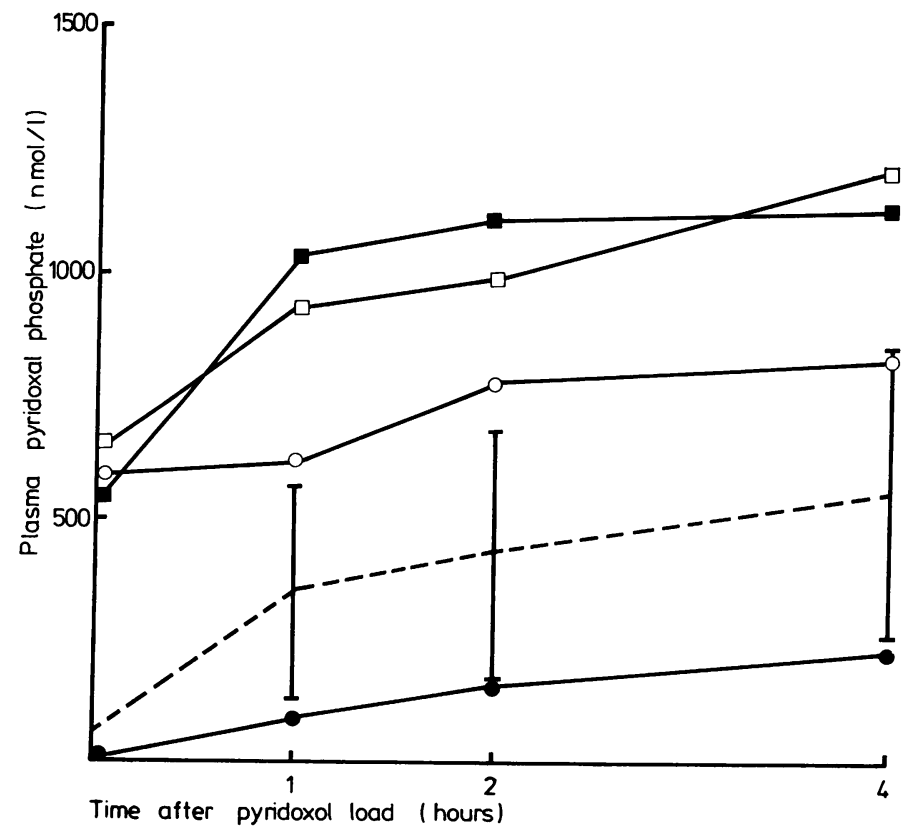

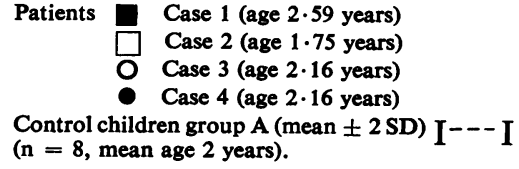

Fig. 3 Plasma pyridoxal phosphate (nmol/l) fasting, and 1, 2, and 4 hours after administration of pyridoxol $\mathrm{HCl}$ orally $(5 \mathrm{mg} / \mathrm{kg})$. 
alternatively, by a greatly increased phosphatase activity. The latter explanation seems less likely in view of the normal 4-PA excretion by the patients.

In the presence of high substrate concentration (POL), as might be expected to occur in hepatocytes after POL loading, an abnormal binding of PALP to albumin might be expected progressively to reduce the rate of POLP oxidation, since pyridoxine kinase activity is subject to product (PALP) inhibition (Wada and Snell, 1961). POL and POLP would accumulate, and as the latter is not protein-bound, it would be susceptible to hydrolysis by phosphatase. This would result in an increased urinary excretion of POL. Three of the 4 patients investigated were found to have significantly raised urinary excretions of POL after oral loading.

The normal outcome of tests-such as tryptophan loading and erythrocyte aminotransferase activitywhich assess the metabolic availability of PALP, have been largely responsible for emphasis being placed on a specific abnormality of cerebral metabolism as the most likely cause of vitamin B6 responsive convulsions in early infancy. The proposed abnormality of albumin binding of PALP in this disorder is not incompatible with such normal findings. It has been demonstrated that these patients do not lack the ability to phosphorylate POL, and therefore, PALP would be available for binding to the cofactor sites of hepatocyte and erythrocyte enzymes. A greater metabolic significance of the proposed defect is predicted for an organ such as brain, which possesses a moderate blood-brain barrier for the vitamin, and for which there is experimental evidence to suggest that an extracerebral compartment is involved in maintaining a constant supply of PALP in that tissue.

The definite abnormal response to oral POL loading when Cases 1-4 were first investigated, and the normal results when they were retested more than one year later, are difficult to reconcile with the notion of a primary inborn error of metabolism, and also with the patients' continuing requirements for large amounts of POL in their clinical management. Scriver and Cullen (1965) first pointed out that an age-related factor might be responsible for modifying a previously-described abnormal excretion of vitamin B6 metabolites in this condition. However, direct comparison between the results of the present and previous investigations (Coursin, 1960; Scriver, 1960) is not possible because, apart from 4-PA excretion, different parameters of vitamin B6 metabolism were studied.

The evidence for an inborn error of glutamic acid metabolism in this disorder of early infancy is open to criticism. One patient has been reported to have had negligible activity of glutamic acid decarboxylase activity when measured in renal biopsy material (Yoshida et al., 1971). However, remarkable differences in activity between kidney and brain decarboxylases have been demonstrated (Haber et al., 1970). It is, therefore, apparent that studies on the brain enzyme are essential to determine whether or not a primary defect in its activity occurs in B6 dependency. While it is recognised that much of the interpretation given to the results of the present investigation is purely speculative, in the absence of definitive evidence for abnormal glutamic acid metabolism in this condition, it is suggested that an alternative pathology is worthy of further investigation.

We are grateful to the physicians and surgeons at The Hospital for Sick Children for permission to study their patients. Financial assistance was provided by the Nuffield Foundation and by the Joint Research Board of the Hospitals for Sick Children and the Institute of Child Health.

\section{References}

Anderson, J. A., Chang, H. W., and Grandjean, C. J. (1971). Nature of the binding site of pyridoxal 5 '-phosphate to bovine serum albumin. Biochemistry, 10, 2408-2415.

Bonavita, V. (1960). The reaction of pyridoxal 5-phosphate with cyanide and its analytical use. Archives of Biochemistry and Biophysics, 88, 366-372.

Chabner, B., and Livingstone, D. (1970). A simple enzymic assay for pyridoxal phosphate. Analytical Biochemistry, 34, 413-423.

Colombini, C. E., and McCoy, E. E. (1970). Vitamin B6 metabolism. The utilization of $\left[{ }^{14} \mathrm{C}\right]$ pyridoxine by the normal mouse. Biochemistry, 9, 533-538.

Coursin, D. B. (1960). Seizures in vitamin B6 deficiency. In Inhibition in the Nervous System and Gamma-aminobutyric Acid, pp. 294-301. Edited by E. Roberts. Pergamon Press: New York.

Coursin, D. B. (1964). Vitamin B6 metabolism in infants and children. Vitamins and Hormones, 22, 755-786.

Dempsey, W. B., and Christensen, H. N. (1962). The specific binding of pyridoxal 5'-phosphate to bovine plasma albumin. Journal of Biological Chemistry, 237, 1113-1120.

Gentz, J., Hamfelt, A., Johansson, S., Lindstedt, S., Persson, B., and Zetterström, R. (1967). Vitamin B6 metabolism in pyridoxine dependency with seizures. Acta paediatrica Scandinavica, 56, 17-26.

Haber, B., Kuriyama, K., and Roberts, E. (1970). An anion stimulated L-glutamic acid decarboxylase in non-neural tissues. Biochemical Pharmacology, 19, 1119-1136.

Hamfelt, A. (1966). The effect of pyridoxal phosphate on the aminotransferase assay in blood. Scandinavian Journal of Clinical and Laboratory Investigation, 18, Supplement 92, 181-188.

Hunt, A. D., Jr, Stokes, J., McCrory, W. W., and Stroud, H. H. (1954). Pyridoxine dependency: report of a case of intractable convulsions in an infant controlled by pyridoxine. Pediatrics, 13, 140-145.

Johansson, M. D., Lindstedt, S., Register, U., and Wadström, L. (1966). Studies on the metabolism of labelled pyridoxine in man. American Journal of Clinical Nutrition, 18, 185-196. 
Johansson, S., Lindstedt, S., and Tiselius, H-G. (1968). Metabolism of $\left[{ }^{3} \mathrm{H}_{8}\right]$ pyridoxine in mice. Biochemistry, 7, 2327-2332.

Johansson, S., Lindstedt, S., and Tiselius, H. G. (1974). Metabolic interconversions of different forms of vitamin B6. Journal of Biological Chemistry, 249, 6040-6046.

Lumeng, L., Brashear, R. E., and Li, T. K. (1974). Pyridoxal 5 '-phosphate in plasma: source, protein-binding, and cellular transport. Journal of Laboratory and Clinical Medicine, 84, 334-343.

Mudd, S. H. (1971). Pyridoxine-responsive genetic disease. Federation Proceedings, 30, 970-976.

Rabinowitz, J. C., and Snell, E. E. (1949). Vitamin B6 group. XV. Urinary excretion of pyridoxal, pyridoxamine, pyridoxine, and 4-pyridoxic acid in human subjects. Proceedings of the Society for Experimental Biology and Medicine, 70, 235-240.

Reddy, S. K., Reynolds, M. S., and Price, J. M. (1958). The determination of 4-pyridoxic acid in human urine. Journal of Biological Chemistry, 233, 691-696.

Scriver, C. R. (1960). Vitamin B6-dependency and infantile convulsions. Pediatrics, 26, 62-74.

Scriver, C. R., and Cullen, A. M. (1965). Urinary vitamin B6 and 4-pyridoxic acid in health and in vitamin B6dependency. Pediatrics, 36, 14-20.

Scriver, C. R., and Whelan, D. T. (1969). Glutamic acid decarboxylase in mammalian tissue outside the central nervous system, and its possible relevance to hereditary vitamin B6-dependency with seizures. Annals of the New York Academy of Sciences, 166, 83-96.

Storvick, C. A., Benson, E. M., Edwards, M. A., and Woodring, M. J. (1964). Chemical and microbiological determination of vitamin B6. In Methods of Biochemical Analysis, volume 12, pp. 210, 212, 255. Edited by D. Glick. Wiley Interscience: New York.

Tiselius, H-G. (1973). Metabolism of tritium-labelled pyridoxine and pyridoxine $5^{\prime}$-phosphate in the central nervous system. Journal of Neurochemistry, 20, 937-946.

Wada, H., and Snell, E. E. (1961). The enzymatic oxidation of pyridoxine and pyridoxamine phosphates. Journal of Biological Chemistry, 236, 2089-2095.

Willey, P. (1972). EEG studies in three infants with pyridoxine dependency. Journal of the Electro-Physiological Technologists Association, 19, 3-13.

Yoshida, T., Tada, K., and Arakawa, T. (1971). Vitamin B6-dependency of glutamic acid decarboxylase in the kidney from a patient with vitamin B6-dependent convulsion. Tohoku Journal of Experimental Medicine, 104, 195-198.

Correspondence to Professor Barbara Clayton, Department of Chemical Pathology, The Hospital for Sick Children, Great Ormond Street, London WC1N 3JH.

The following articles will appear in future issues of this journal:

Evaluation of methods of assisted ventilation in hyaline membrane disease. F. P. Manginello, A. E. Grassi, S. Schechner, A. N. Krauss, and P. A. M. Auld.

Serum theophylline levels in asthmatic children after oral administration of two slow-release theophylline preparations. S. McKenzie and E. Baillie.

Improved accuracy of lactose tolerance test in children, using expired $\mathrm{H}_{2}$ measurement. $A$. C. Douwes, J. Fernandes, and H. J. Degenhart.

Mineral and trace metal supplement for use with synthetic diets based on comminuted chicken. $J . M$. Thorn, P. J. Aggett, H. T. Delves, and B. E. Clayton.

A case of paragonimiasis. A. Demetriou, B. M. Phillips, and R. G. Hendrickse.

Heart failure apparently due to overfeeding in a neonate. F. Eyal, C. Maayan, and S. Godfrey. 